\title{
The Infinite Time Limit for the Time-Dependent Born-Oppenheimer Approximation
}

\author{
Armin Kargol ${ }^{\star, \star \star}$ \\ Center for Transport Theory and Mathematical Physics, Virginia Polytechnic Institute and State \\ University, Blacksburg, Va 24061, USA. E-mail:kargol@math.vt.edu
}

Received: 6 August 1993 / in revised form: 31 January 1994

\begin{abstract}
We analyze the Schrödinger equation $i \varepsilon^{2} \frac{\partial}{\partial t} \Psi=H(\varepsilon) \Psi$, where $H(\varepsilon)$ is the hamiltonian of the molecular system consisting of nuclei with masses proportional to $\varepsilon^{-4}$ and electrons with masses of order 1. Using the Born-Oppenheimer method we construct the leading order asymptotic expansion to the exact solutions of the equation. We show that if the particles interact through smooth potentials decaying suitably as the distance between particles tends to $\infty$, then the expansion holds uniformly for all times $t \in[0, \infty)$. By similar analysis one can show validity of the expansion for $t \in(-\infty, 0]$, thus our results hold for scattering theory.
\end{abstract}

\section{Introduction}

This work is devoted to the analysis of the dynamics of molecular systems. By molecular systems we mean quantum mechanical systems consisting of two types of particles: heavy (further referred to as nuclei) and light (electrons). It is known that for such systems the task of solving the Schrödinger equation becomes quite difficult, one of the reasons being the large number of particles involved. Therefore one usually resorts to an approximation.

In $1927 \mathrm{M}$. Born and R. Oppenheimer proposed an approximate method of solving the time-independent Schrödinger equation. It proved to be very useful and became known as the Born-Oppenheimer approximation. The main idea was to exploit the disparity between masses of nuclei and electrons. Born and Oppenheimer postulated to use the fourth root of the ratio of electronic mass to nuclear mass as a small parameter $\varepsilon$ and seek approximate energy levels and eigenfunctions of the molecular hamiltonian in forms of power series in $\varepsilon$. However, their calculations were only formal and, quite surprisingly, there was very little rigorous work done on this subject until late 1970's. Then Combes, Duclos, Seiler [1-3] and later Hagedorn $[8,9]$ proved that the Born-Oppenheimer series is asymptotic to the exact solution of the Schrödinger equation to arbitrary order in $\varepsilon$. Recently Klein et al. [11] showed similar result for polyatomic molecules.

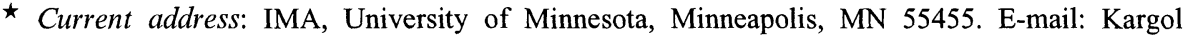
@ima.umn.edu

$\star \star$ The material in this paper is contained in a dissertation submitted to the faculty of VPI \& SU in partial fulfillment of the requirements for the Ph.D. degree.
} 
The time-dependent Born-Oppenheimer approximation, which describes the dynamics of molecular systems, rather than energy levels, has been first rigorously formulated by Hagedorn [6] in 1980. The principal idea is similar to the timeindependent case. Because of the disparity in masses, the electrons move much more rapidly than nuclei. For short times we can approximately find their motion by treating nuclei as fixed. On a longer time scale the electrons quickly adjust their motion to slowly varying position of the nuclei. Therefore we can use the adiabatic approximation to describe the dynamics of electrons. On the other hand the electrons generate an effective potential in which the nuclei, because of their large mass move semiclassically. These two approximations, adiabatic for electrons and semiclassical for nuclei, are however coupled to each other. In order to separate them we use so-called multiple scales technique, which will be described later.

Using this method and a certain semiclassical technique [5], Hagedorn [7] analyzed the dynamics of molecular systems with smooth potentials, on finite time interval $[0, T]$. He proved that solutions to the time-dependent Schrödinger equation have asymptotic expansions of arbitrary order in $\varepsilon$, but his estimates depend on $T$ and therefore cannot be used to analyze scattering problems.

In this work we extend the ideas of Hagedorn [7] to show that the leading order asymptotic expansion obtained in such way is in fact uniform in time for $t \in[0, \infty)$. We restrict our attention to diatomic molecules and require potentials and their derivatives to decay sufficiently fast as the distance between particles increases to $\infty$. By mimicking our proofs one can show uniformity of the expansion for $t \in(-\infty, 0]$. Therefore our results justify use of this expansion in treating scattering problems.

The case of Coulomb potentials has been investigated by Hagedorn [10]. He proved a result similar to the smooth case, but again, for finite times only. We plan to analyze this problem in the context of scattering theory in the near future.

Finally, we mention the work by Klein, Martinez and Wang [12], who analyzed the Born-Oppenheimer approximation for wave operators. They also consider a diatomic molecule with smooth, short-range potentials. Their technique is, however, very different from ours since they consider the time-independent scattering theory.

This paper is organized as follows: in Sect. 2 we specify our choice of coordinate system and introduce necessary notation. We also define so-called semiclassical wave packets which we later use to describe the motion of nuclei. In Sect. 3 we investigate the asymptotic behavior of the eigenvalues of the electronic hamiltonian for small $\varepsilon$ and large intercluster distance. Our main theorem is stated in Sect. 4. This section also describes the multiple scales technique which allows us to decouple the semiclassical and adiabatic aspects of our problem. Finally, the last section contains the rigorous proof of the theorem.

\section{Notation}

We consider a diatomic molecule with $N$ electrons. The masses of nuclei are $\varepsilon^{-4}$ and the masses of electrons are 1 (we rescale the coordinates if necessary). We want to analyze the particular scattering channel where the molecule splits into two clusters each consisting of a nucleus and a certain number of electrons. For this cluster decomposition $\left\{C_{1}, C_{2}\right\}$, where $C_{1}=\underbrace{\left\{1, \ldots, K^{(1)}+1\right\}}$, 
$C_{2}=\underbrace{\left\{K^{(1)}+2, \ldots, N+2\right\}}_{K^{(2)}=N-K^{(1)} \text { electrons, } 1 \text { nucleus }}$, we introduce the clustered Jacobi coordinates [13]. Indices $1, K^{(1)}+2$ refer to the nuclei. Indices $2, \ldots, K^{(1)}+1, K^{(1)}+3, \ldots, N+2$ refer to the electrons. Then $\xi_{1}^{(1)}, \ldots, \xi_{K^{(1)}}^{(1)}, \xi_{1}^{(2)}, \ldots, \xi_{K^{(2)}}^{(2)}$ are the internal coordinates for the clusters (the upper index refers to the cluster), $\zeta$ is the coordinate between the centers of mass of the clusters, and $\mathrm{R}$ is the total center of mass coordinate. In particular we choose $\xi$ 's so that $\xi_{1}^{(1)}, \ldots, \xi_{K^{(1)}-1}^{(1)}$, (resp. $\xi_{1}^{(2)}, \ldots, \xi_{K^{(2)}-1}^{(2)}$ ) involve only electrons and $\xi_{K^{(1)}}^{(1)}$, (resp. $\xi_{K^{(2)}}^{(2)}$ ) is the vector from the center of mass of all electrons in given cluster to the nucleus of the cluster (see Fig. 1). This choice simplifies the dependence of the potentials on $\varepsilon$ (see the comment following formula (2c) below).

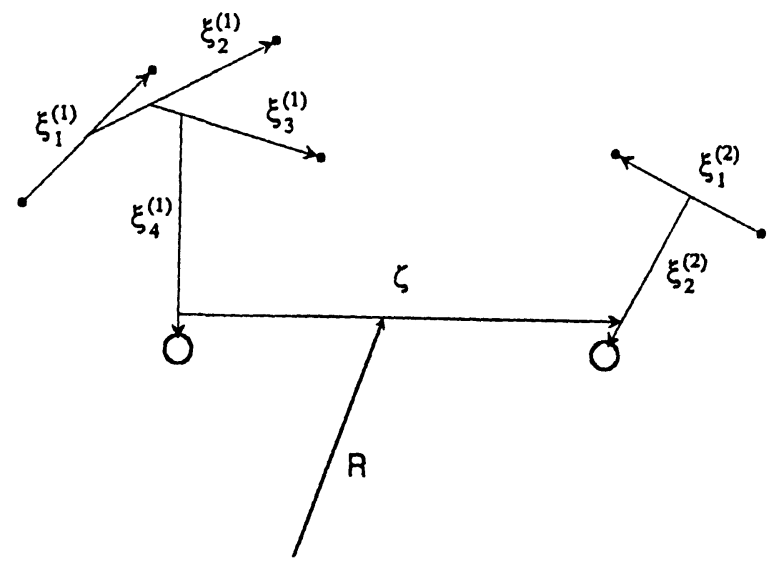

Fig. 1. An example of the clustered Jacobi coordinates $\left(N=6, K^{(1)}=4, K^{(2)}=2\right)$. electron; $\bigcirc$ nucleus

In these coordinates the hamiltonian becomes:

$$
\tilde{H}=-\frac{1}{2 \varepsilon^{-4}+N} \Delta_{R}-\frac{\varepsilon^{4}}{2} \mu(\varepsilon) \Delta_{\zeta}-\sum_{i=1}^{K^{(1)}} \frac{v_{i}^{(1)}(\varepsilon)}{2} \Delta_{\xi_{i}^{(1)}}-\sum_{i=1}^{K^{(2)}} \frac{v_{i}^{(2)}(\varepsilon)}{2} \Delta_{\xi_{i}^{(2)}}+V,
$$

where $\mu(\varepsilon)$ and $v_{i}^{(j)}(\varepsilon)$ are rational functions of $\varepsilon^{4}$ tending to nonzero constants as $\varepsilon \rightarrow 0$.

We remove the center of mass motion and rescale $\zeta$, so that:

$$
H=-\frac{\varepsilon^{4}}{2} \Delta_{\zeta}-\sum_{i=1}^{K^{(1)}} \frac{v_{i}^{(1)}(\varepsilon)}{2} \Delta_{\xi_{I}^{(1)}}-\sum_{i=1}^{K^{(2)}} \frac{v_{i}^{(2)}(\varepsilon)}{2} \Delta_{\xi_{i}^{(2)}}+V
$$

where:

$$
V=V^{\left(C_{1}\right)}+V^{\left(C_{2}\right)}+V_{D, \varepsilon} .
$$

$V^{\left(C_{J}\right)}$ is the interaction within $\mathrm{j}$-th cluster, $V_{D, \varepsilon}$ is the intercluster interaction. Explicitly: 


$$
\begin{aligned}
V^{\left(C_{1}\right)} & =\sum_{2 \leqq i<j \leqq K^{(1)}+1} V_{i j}\left(\sum_{k=1}^{K^{(1)}-1} \mu_{k}^{(1)} \xi_{k}^{(1)}\right)+\sum_{i=2}^{K^{(1)}+1} V_{i, 1}\left(\sum_{k=1}^{K^{(1)}} \tilde{\mu}_{k}^{(1)} \xi_{k}^{(1)}\right) \\
V^{\left(C_{2}\right)} & =\sum_{K^{(1)}+3 \leqq i<j \leqq N+2} V_{i j}\left(\sum_{k=1}^{K^{(2)}-1} \mu_{k}^{(2)} \xi_{k}^{(2)}\right)+\sum_{i=K^{(1)}+3}^{N+2} V_{i, K^{(1)}+2}\left(\sum_{k=1}^{K^{(2)}} \tilde{\mu}_{k}^{(2)} \xi_{k}^{(2)}\right),(2) \\
V_{D, \varepsilon} & =\sum_{i \in C_{1}, j \in C_{2}} V_{i j}\left(\tau(\varepsilon) \zeta+\sum_{l=1}^{2} \sum_{k=1}^{K^{(l)}} \tau_{k}^{(l)}(\varepsilon) \xi_{k}^{(l)}\right)
\end{aligned}
$$

Here $\tau_{k}^{(l)}, \mu_{k}^{(l)}$, and $\tilde{\mu}_{k}^{(l)}$ obviously depend on $i, j$ and our choice of Jacobi coordinates. One can also see that $\tau_{k}^{(l)}$ equal zero or constants for $k=1, \ldots, K^{(l)}-1 . \tau_{K^{(l)}}^{(l)}$ are rational functions of $\varepsilon^{4}$ that approach constants as $\varepsilon \rightarrow 0$ or tend to 0 like $\varepsilon^{4}$. $\mu_{k}^{(l)}$ and $\tilde{\mu}_{k}^{(l)}$ are $\varepsilon$-independent.

$V_{i j}$ are the two body potentials depending only on the relative positions of the interacting particles. We also assume that they are at least $C^{3}$ and decay at infinity according to:

$$
\begin{aligned}
&|V(x)| \leqq C_{1}(1+|x|)^{-1-\delta}, \\
&\left|\partial_{x^{i}} V(x)\right| \leqq C_{2}(1+|x|)^{-2-\delta}, \\
&\left|\partial_{x^{i}} \partial_{x^{\prime}} V(x)\right| \leqq C_{3}(1+|x|)^{-3-\delta}, \\
&\left|\partial_{x^{i}} \partial_{x^{\prime}} \partial_{x^{k}} V(x)\right| \leqq C_{4}(1+|x|)^{-4-\delta},
\end{aligned}
$$

for some constants $C_{1}, \ldots, C_{4}$ and $\delta>0$. We will use the following notation:

$$
\begin{aligned}
& h_{D}^{(1)}(\varepsilon)=-\frac{1}{2} \sum_{i=1}^{K^{(1)}} v_{i}^{(1)}(\varepsilon) \Delta_{\xi_{i}(1)}+V^{\left(C_{1}\right)} \\
& h_{D}^{(2)}(\varepsilon)=-\frac{1}{2} \sum_{i=1}^{K^{(2)}} v_{i}^{(2)}(\varepsilon) \Delta_{\xi_{i}^{(2)}}+V^{\left(C_{2}\right)}
\end{aligned}
$$

Then we can write the hamiltonian $H$ as a sum of nuclear kinetic part $-\frac{\varepsilon^{4}}{2} \Delta_{\zeta}$ and so-called electronic hamiltonian $h_{\varepsilon}(\zeta)$, where:

$$
\begin{aligned}
h_{\varepsilon}(\zeta)= & h_{D}^{(1)}(0)+h_{D}^{(2)}(0)-\frac{1}{2} \sum_{l=1}^{2} \sum_{k=1}^{K^{(l)}}\left(v_{k}^{(l)}(\varepsilon)-v_{k}^{(l)}(0)\right) \Delta_{\xi_{k}^{(l)}} \\
& +V_{D, \varepsilon}\left(\zeta, \xi^{(1)}, \xi^{(2)}\right) \\
= & h_{D}+\varepsilon^{4} D(\varepsilon)+V_{D, \varepsilon}\left(\zeta, \xi^{(1)}, \xi^{(2)}\right) .
\end{aligned}
$$


Here we used the obvious abbreviation:

$$
\begin{aligned}
h_{D} & =h_{D}^{(1)}(0)+h_{D}^{(2)}(0), \\
\varepsilon^{4} D(\varepsilon) & =-\frac{1}{2} \sum_{l=1}^{2} \sum_{k=1}^{K^{(l)}}\left(v_{k}^{(l)}(\varepsilon)-v_{k}^{(l)}(0)\right) \Delta_{\xi_{k}}, \\
\xi & =\left(\xi^{(1)}, \xi^{(2)}\right), \\
\xi^{(1)} & =\left(\xi_{1}^{(1)}, \ldots, \xi_{K^{(1)}}\right), \\
\xi^{(2)} & =\left(\xi_{1}^{(2)}, \ldots, \xi_{K^{(2)}}\right) .
\end{aligned}
$$

To complete the notation we define so-called semiclassical wave packets [5], which will be used later to describe the motion of nuclei in the potential given by $E(x)$.

Definition. Let $A, B$ be complex $3 \times 3$ matrices such that:

$A$ and $B$ are invertible,

$$
B A^{-1} \text { is symmetric, }
$$

$$
\begin{gathered}
\operatorname{Re} B A^{-1}=\frac{1}{2}\left[B A^{-1}+\left(B A^{-1}\right)^{*}\right] \text { is strictly positive definite, } \\
\left(\operatorname{Re} B A^{-1}\right)^{-1}=A A^{*} .
\end{gathered}
$$

Let $a \in \mathbb{R}^{3}, \eta \in \mathbb{R}^{3}, \varepsilon>0$. Then we define:

$$
\begin{aligned}
& \varphi\left(A, B, \varepsilon^{2}, a, \eta, x\right):= \\
& \pi^{-\frac{3}{4}} \varepsilon^{-\frac{3}{2}}(\operatorname{det} A)^{-\frac{1}{2}} \exp \left(-\frac{\left\langle(x-a), B A^{-1}(x-a)\right\rangle}{2 \varepsilon^{2}}+i \frac{\langle\eta,(x-a)\rangle}{\varepsilon^{2}}\right) .
\end{aligned}
$$

Let $A_{+}, B_{+}$be such matrices and $a_{+}, \eta_{+} \in \mathbb{R}^{3}, \eta_{+} \neq 0$. Consider the system of equations:

$$
\begin{aligned}
& \frac{d \eta(t)}{d t}=-E^{\prime}(a(t)), \\
& \frac{d a(t)}{d t}=\eta(t), \\
& \frac{d A(t)}{d t}=i B(t), \\
& \frac{d B(t)}{d t}=i E^{\prime \prime}(a(t)) A(t),
\end{aligned}
$$

where $E^{\prime}$ denotes the gradient of $E$ and $E^{\prime \prime}$ is the matrix of second derivatives of $E$.

One can show [5] that if $E(x)$ satisfies conditions (3) then there is a unique solution $a(t), \eta(t), A(t), B(t)$ to (9) satisfying the following conditions:

$$
\begin{gathered}
\lim _{t \rightarrow \infty}\left\|A(t)-A_{+}-i B_{+} t\right\|=0, \\
\lim _{t \rightarrow \infty}\left\|B(t)-B_{+}\right\|=0,
\end{gathered}
$$




$$
\begin{gathered}
\lim _{t \rightarrow \infty}\left|a(t)-a_{+}-\eta_{+} t\right|=0, \\
\lim _{t \rightarrow \infty}\left|\eta(t)-\eta_{+}\right|=0 .
\end{gathered}
$$

Finally we define:

$$
S(t)=\int_{0}^{t}\left(\frac{\eta(s)^{2}}{2}-E(a(s))\right) d s .
$$

Here the first two equations $(9 \mathrm{a}, \mathrm{b})$ are just the Newton's equations with $a(t)$ being the classical path and $\eta(t)$ - the classical momentum. Matrices $A(t)$ and $B(t)$ describe "spreading" of the wave packet in position and momentum space. Conditions (10) mean that in the limit $t \rightarrow \infty$ the dynamics of the interacting clusters approaches that of the noninteracting ones.

\section{Properties of the Electronic Hamiltonian}

Usually the electronic hamiltonian is defined by formal substitution $\varepsilon=0$ in (1). Then it becomes an operator acting on functions of "electronic" coordinates $\xi$, depending parametrically on the intercluster distance $\zeta$. Our choice is slightly different. We allow certain $\varepsilon$-dependence in the electronic hamiltonian, which we denote by a subscript, as in $h_{\varepsilon}(\zeta)$ defined by (6). This is the result of our choice of coordinates. By using the Jacobi coordinates we avoided so-called Hughes-Eckart terms in the kinetic part, but we have to deal with $\varepsilon$-dependence of both the kinetic part of the electronic hamiltonian and the intercluster interaction. The dependence of the reduced masses $v_{k}^{(i)}$ on $\varepsilon$ can be easily taken care of. One can see that $D(\varepsilon)$ is relatively bounded with respect to $h_{D}$ and therefore it is a regular perturbation. It causes the eigenvalues and eigenvectors of $h_{D}+\varepsilon^{4} D(\varepsilon)+V_{D, \varepsilon}(\zeta, \xi)$ to be smooth functions of $\varepsilon^{4}$.

Unfortunately $\varepsilon$ also appears in the argument of the intercluster interaction. The rest of this section is devoted to the analysis of behavior of the eigenvalues $E_{\varepsilon}(\zeta)$ for large $\zeta$ and small $\varepsilon$.

We assume that $h_{D}$ has a simple isolated eigenvalue $E_{D}$ with eigenvector $\varphi_{D}(\xi)$ and that this eigenvalue is stable, i.e. there is a unique simple isolated eigenvalue $E_{\varepsilon}(\zeta)$ of $h_{\varepsilon}(\zeta)$ which tends to $E_{D}$ as $|\zeta| \rightarrow \infty$ and $\varepsilon \rightarrow 0$. We denote the corresponding eigenvector by $\varphi_{\varepsilon}(\xi, \zeta)$.

In the following we assume that $|\zeta|$ is large enough and $\varepsilon$ small enough, so we can find $\gamma>0$ such that $E_{D}$ and $E_{\varepsilon}(\zeta)$ lie inside the circle $\left|z-E_{D}\right|=\frac{\gamma}{2}$ and there is no other eigenvalue in $\left|z-E_{D}\right|=\gamma$.

Lemma 1. Assume that the potentials satisfy (3). Let $P_{\varepsilon}(\zeta)$ be the projection onto the eigenvector $\varphi_{\varepsilon}(\xi, \zeta)$ associated with the eigenvalue $E_{\varepsilon}(\zeta)$ of $h_{\varepsilon}(\zeta)$. Then:

$$
\begin{gathered}
\left\|\partial_{\zeta^{l}} P_{\varepsilon}(\zeta) \varphi_{D}(\xi)\right\|_{L^{2}\left(\mathbb{R}^{3 N}, d \xi\right)} \leqq C_{1}(1+|\zeta|)^{-2-\delta} \\
\left\|\partial_{\zeta^{i}} \partial_{\zeta^{j}} P_{\varepsilon}(\zeta) \varphi_{D}(\xi)\right\|_{L^{2}\left(\mathbb{R}^{3 N}, d \xi\right)} \leqq C_{2}(1+|\zeta|)^{-3-\delta} \\
\left\|\partial_{\zeta^{\iota}} \partial_{\zeta^{J}} \partial_{\zeta^{k}} P_{\varepsilon}(\zeta) \varphi_{D}(\xi)\right\|_{L^{2}\left(\mathbb{R}^{3 N}, d \xi\right)} \leqq C_{3}(1+|\zeta|)^{-4-\delta},
\end{gathered}
$$

uniformly for small $\varepsilon$, where $\delta$ is the same constant as in (3). 
Proof. a) From:

$$
\partial_{\zeta^{l}} P_{\varepsilon}(\zeta) \varphi_{D}=\frac{1}{2 \pi i} \int_{\left|z-E_{D}\right|=\gamma}\left(h_{\varepsilon}(\zeta)-z\right)^{-1}\left(\partial_{\zeta^{i}} V_{D, \varepsilon}\right)\left(h_{\varepsilon}(\zeta)-z\right)^{-1} \varphi_{D} d z
$$

we get:

$$
\begin{aligned}
&\left\|\partial_{\zeta^{l}} P_{\varepsilon}(\zeta) \varphi_{D}\right\|_{L^{2}\left(\mathbb{R}^{3 N}, d \xi\right)} \leqq C_{4} \int_{\left|z-E_{D}\right|=\gamma}\left\|\left(h_{\varepsilon}(\zeta)-z\right)^{-1}\right\|_{\text {op }}\left\|\left(\partial_{\zeta^{i}} V_{D, \varepsilon}\right) g\right\|_{L^{2}\left(\mathbb{R}^{3 N}, d \xi\right)} d z \\
& \leqq C_{5} \sup _{\left|z-E_{D}\right|=\gamma}\left(\left\|\left(h_{\varepsilon}(\zeta)-z\right)^{-1}\right\|\left\|_{\text {op }}\right\|\left(\partial_{\zeta^{i}} V_{D, \varepsilon}\right) g \|_{L^{2}\left(\mathbb{R}^{3 N}, d \xi\right)}\right),
\end{aligned}
$$

where $g(\xi):=\left(h_{\varepsilon}(\zeta)-z\right)^{-1} \varphi_{D}(\xi)$. Note that $\sup _{\left|z-E_{D}\right|=\gamma}\left(\left\|\left(h_{\varepsilon}(\zeta)-z\right)^{-1}\right\|_{\text {op }}\right)$ is bounded uniformly in $\zeta$ for large $\zeta$ by our assumption on $E_{\varepsilon}(\zeta)$.

Therefore, conclusion (12a) will be proved if we can show:

$$
\left\|\left(\partial_{\zeta^{i}} V_{D, \varepsilon}\right) g\right\|_{L^{2}\left(\mathbb{R}^{3 N}, d \xi\right)} \leqq C_{6}(1+|\zeta|)^{-2-\delta} .
$$

The eigenvector $\varphi_{D}$ decays exponentially so it is in the weighted $L^{2}$ space $\left(L_{\mu}^{2}\right)$ for arbitrary $\mu$ (i.e. $\left.\left\|\varphi_{D}\right\|_{\mu}:=\left\|\left(1+|\xi|^{2}\right)^{\frac{\mu}{2}} \varphi_{D}(\xi)\right\|_{L^{2}(d \xi)}<\infty\right)$. It is proved [14] (see Appendix, Lemma A) that, for suitable potentials, $(-\Delta+V-z)^{-1}$ is a bounded operator from $L_{\mu}^{2}$ to $L_{\mu}^{2}$. It follows that $g(\xi)$ is in $L_{\mu}^{2}$ for any $\mu$. In particular:

$$
\left\|\left(1+\left|\xi_{1}^{(1)}\right|^{2}\right)^{\frac{\mu}{2}} \ldots\left(1+\left|\xi_{K^{(2)}}^{(2)}\right|^{2}\right)^{\frac{\mu}{2}} g\left(\xi_{1}^{(1)}, \ldots, \xi_{K^{(2)}}^{(2)}\right)\right\|_{L^{2}\left(\mathbb{R}^{3}, d \xi\right)} \leqq C_{7}
$$

for any $\mu>0$. Moreover, the constant $C_{7}$ is $\zeta$-independent.

In order to estimate $\left\|\left(\partial_{\zeta^{l}} V_{D, \varepsilon}\right) g\right\|_{L^{2}\left(\mathbb{R}^{3 N}, d \xi\right)}$ we split $\partial_{\zeta^{i}} V_{D, \varepsilon}$ according to (2c). We show calculations for the term where all $\tau_{j}^{\prime} s$ are nonzero. In other terms if one or more of $\tau_{j}^{\prime} s$ are zero then the corresponding variables $\xi_{j}^{(k)}$ appear only in $g$. For example, if $\tau_{1}^{(1)}=0$, then we can do the integral with respect to $\xi_{1}^{(1)}$. Note that

$$
g^{1}\left(\xi_{2}^{(1)}, \ldots, \xi_{K^{(2)}}^{(2)}\right):=\left[\int\left|g\left(\xi_{1}^{(1)}, \xi_{2}^{(1)}, \ldots, \xi_{K^{(2)}}^{(2)}\right)\right|^{2} d^{3} \xi_{1}^{(1)}\right]^{\frac{1}{2}}
$$

is in $L_{\mu}^{2}\left(\mathbb{R}^{3 N-3}, d \xi_{2}^{(1)} \ldots \xi_{K^{(2)}}^{(2)}\right)$. This way we eliminate all the coordinates $\xi_{j}^{(k)}$ for which $\tau_{j}^{(k)}=0$ and we are back to the general situation.

Hence we need to estimate:

$$
\left\|\partial_{\zeta_{l}} V_{m n}\left(\tau(\varepsilon) \zeta+\sum_{k=1}^{K^{(1)}} \tau_{k}^{(1)}(\varepsilon) \xi_{k}^{(1)}+\sum_{k=1}^{K^{(2)}} \tau_{k}^{(2)}(\varepsilon) \xi_{k}^{(2)}\right) \cdot g\left(\xi^{(1)}, \xi^{(2)}\right)\right\|_{L^{2}\left(\mathbb{R}^{3 N}, d \xi\right)} .
$$

We separate $\xi_{1}^{(1)}$ in the argument of $\partial_{\zeta_{l}} V_{m n}$ and call the rest $u_{1}$, i.e.:

$$
u_{1}=\tau(\varepsilon) \zeta+\sum_{k=2}^{K^{(1)}} \tau_{k}^{(1)}(\varepsilon) \xi_{k}^{(1)}+\sum_{k=1}^{K^{(2)}} \tau_{k}^{(2)}(\varepsilon) \xi_{k}^{(2)}
$$

and do the $\xi_{1}^{(1)}$ integral in (13). Let:

$$
\begin{aligned}
& B_{1}^{1}=\left\{y:\left|u_{1}+y\right| \leqq \kappa\left|u_{1}\right|\right\}, \\
& B_{2}^{1}=\left\{y:\left|u_{1}+y\right|>\kappa\left|u_{1}\right|\right\}
\end{aligned}
$$


for some $0<\kappa<1$, and let $\chi_{1}^{1}, \chi_{2}^{1}$ be the corresponding characteristic functions. Then:

$$
\begin{aligned}
& \left\|\left(\partial_{\zeta^{i}} V_{m n}\left(u_{1}+\tau_{1}^{(1)}(\varepsilon) \xi_{1}^{(1)}\right)\right) \cdot g(\xi)\right\|_{L^{2}\left(\mathbb{R}^{3}, d \xi_{1}^{(1)}\right)} \\
& =\tau_{1}^{(1)}(\varepsilon)^{-\frac{3}{2}}\left\|\chi_{1}^{1}\left(y_{1}\right)\left(\partial_{\zeta^{i}} V_{m n}\left(u_{1}+y_{1}\right)\right) \cdot g\left(\tau_{1}^{(1)}(\varepsilon)^{-1} y_{1}, \xi_{2}^{(1)}, \ldots, \xi_{K^{(2)}}^{(2)}\right)\right\|_{L^{2}\left(\mathbb{R}^{3}, d y_{1}\right)} \\
& \quad+\tau_{1}^{(1)}(\varepsilon)^{-\frac{3}{2}}\left\|\chi_{2}^{1}\left(y_{1}\right) \cdot\left(\partial_{\zeta^{i}} V_{m n}\left(u_{1}+y_{1}\right)\right) \cdot g\left(\tau_{1}^{(1)}(\varepsilon)^{-1} y_{1}, \xi_{2}^{(1)}, \ldots, \xi_{K^{(2)}}^{(2)}\right)\right\|_{L^{2}\left(\mathbb{R}^{3}, d y_{1}\right)} \\
& =I_{1}\left(u_{1}\right)+I_{2}\left(u_{1}\right) .
\end{aligned}
$$

For $I_{2}\left(u_{1}\right)$ we note:

$$
\begin{aligned}
I_{2}\left(u_{1}\right) \leqq & \tau_{1}^{(1)}(\varepsilon)^{-\frac{3}{2}}\left\|\chi_{2}^{1}\left(y_{1}\right) \partial_{\zeta^{i}} V_{m n}\left(u_{1}+y_{1}\right)\right\|_{\infty} \\
& \times\left\|g\left(\tau_{1}^{(1)}(\varepsilon)^{-1} y_{1}, \xi_{2}^{(1)}, \ldots, \xi_{K^{(2)}}^{(2)}\right)\right\|_{L^{2}\left(\mathbb{R}^{3}, d y_{1}\right)} \\
\leqq & \tau_{1}^{(1)}(\varepsilon)^{-\frac{3}{2}}\left\|\chi_{2}^{1}\left(y_{1}\right) \partial_{\zeta^{i}} V_{m n}\left(u_{1}+y_{1}\right)\right\|_{\infty} \\
& \times\left\|\left(1+\left|\tau_{1}^{(1)}(\varepsilon)^{-1} y_{1}\right|^{2}\right)^{\frac{\mu}{2}} g\left(\tau_{1}^{(1)}(\varepsilon)^{-1} y_{1}, \ldots, \xi_{K^{(2)}}^{(2)}\right)\right\|_{L^{2}\left(\mathbb{R}^{3}, d y_{1}\right)} \\
& \times\left\|\left(1+\left|\tau_{1}^{(1)}(\varepsilon)^{-1} y_{1}\right|^{2}\right)^{-\frac{\mu}{2}}\right\|_{\infty},
\end{aligned}
$$

where $\|\cdot\|_{\infty}$ denotes the $L^{\infty}$ norm in variable $\xi_{1}^{(1)}$. Here $\|(1+$ $\left.\left|\tau_{1}^{(1)}(\varepsilon)^{-1} y_{1}\right|^{2}\right)^{-\frac{\mu}{2}} \|_{\infty}=1$.

Also:

$$
\begin{aligned}
g^{1}\left(\xi_{2}^{(1)}, \ldots, \xi_{K^{(2)}}^{(2)}\right):= & \tau_{1}^{(1)}(\varepsilon)^{-\frac{3}{2}} \|\left(1+\left|\tau_{1}^{(1)}(\varepsilon)^{-1} y_{1}\right|^{2}\right)^{\frac{\mu}{2}} g \\
& \times\left(\tau_{1}^{(1)}(\varepsilon)^{-1} y_{1}, \ldots, \xi_{K^{(2)}}^{(2)}\right) \|_{L^{2}\left(\mathbb{R}^{3}, d y_{1}\right)} \\
= & \left\|\left(1+\left|\xi_{1}^{(1)}\right|^{2}\right)^{\frac{\mu}{2}} g\left(\xi_{1}^{(1)}, \ldots, \xi_{K^{(2)}}^{(2)}\right)\right\|_{L^{2}\left(\mathbb{R}^{3}, d \xi_{1}^{(1)}\right)}
\end{aligned}
$$

is in $L_{\mu}^{2}\left(\mathbb{R}^{3 N-3}, d \xi_{2}^{(1)}, \ldots, \xi_{K^{(2)}}^{(2)}\right)$ and

$$
\left\|\chi_{2}^{1}\left(y_{1}\right)\left(\partial_{\zeta^{l}} V_{m n}\left(u_{1}+y_{1}\right)\right)\right\|_{\infty}=\sup _{y_{1} \in B_{2}}\left|\left(\partial_{\zeta^{i}} V_{m n}\left(u_{1}+y_{1}\right)\right)\right| \leqq C_{8}\left(1+\kappa\left|u_{1}\right|\right)^{-2-\delta}
$$

Then:

$$
I_{2}\left(u_{1}\right) \leqq \frac{C_{8}}{\kappa^{2+\delta}}\left(1+\left|u_{1}\right|\right)^{-2-\delta} g^{1}\left(\xi_{2}^{(1)}, \ldots, \xi_{K^{(2)}}^{(2)}\right)
$$


Now let's concentrate on $I_{1}$ :

$$
\begin{aligned}
I_{1}\left(u_{1}\right)= & \tau_{1}^{(1)}(\varepsilon)^{-\frac{3}{2}}\left\|\chi_{1}^{1}\left(y_{1}\right)\left(\partial_{\xi^{i}} V_{m n}\left(u_{1}+y_{1}\right)\right) g\left(\tau_{1}^{(1)}(\varepsilon)^{-1} y_{1}, \xi_{2}^{(1)}, \ldots, \xi_{K^{(2)}}^{(2)}\right)\right\|_{L^{2}\left(\mathbb{R}^{3}, d y_{1}\right)} \\
\leqq & \tau_{1}^{(1)}(\varepsilon)^{-\frac{3}{2}}\left\|\chi_{1}^{1}\left(y_{1}\right)\left(\partial_{\zeta^{1}} V_{m n}\left(u_{1}+y_{1}\right)\right)\right\|_{\infty} \\
& \times\left\|\chi_{1}^{1}\left(y_{1}\right) g\left(\tau_{1}^{(1)}(\varepsilon)^{-1} y_{1}, \xi_{2}^{(1)}, \ldots, \xi_{K^{(2)}}^{(2)}\right)\right\|_{L^{2}\left(\mathbb{R}^{3}, d y_{1}\right)} \\
\leqq & C_{9} \tau_{1}^{(1)}(\varepsilon)^{-\frac{3}{2}} \| \chi_{1}^{1}\left(y_{1}\right)\left(1+\left|\tau_{1}^{(1)}(\varepsilon) y_{1}\right|^{2}\right)^{-\frac{\mu}{2}}\left(1+\left|\tau_{1}^{(1)}(\varepsilon)^{-1} y_{1}\right|^{2}\right)^{\frac{\mu}{2}} \\
& \times g\left(\tau_{1}^{(1)}(\varepsilon)^{-1} y_{1}, \xi_{2}^{(1)}, \ldots, \xi_{\left.K^{(2)}\right)}^{(2)}\right) \|_{L^{2}\left(\mathbb{R}^{3}, d y_{1}\right)} \\
\leqq & C_{9} \tau_{1}^{(1)}(\varepsilon)^{-\frac{3}{2}}\left\|\chi_{1}^{1}\left(y_{1}\right)\left(1+\left|\tau_{1}^{(1)}(\varepsilon)^{-1} y_{1}\right|^{2}\right)^{-\frac{\mu}{2}}\right\|_{\infty} \\
& \times\left\|\left(1+\left|\tau_{1}^{(1)}(\varepsilon) y_{1}\right|^{2}\right)^{\frac{\mu}{2}} g\left(\tau_{1}^{(1)}(\varepsilon)^{-1} y_{1}, \xi_{2}^{(1)}, \ldots, \xi_{\left.K^{(2)}\right)}^{(2)}\right)\right\|_{L^{2}\left(\mathbb{R}^{3}, d y_{1}\right)} \\
\leqq & C_{10}\left(1+\tau_{1}^{(1)}(\varepsilon)^{-2}\left|u_{1}\right|^{2}\left(1-\kappa^{2}\right)\right)^{-\frac{\mu}{2}} g^{1}\left(\xi_{2}^{(1)}, \ldots, \xi_{K^{(2)}}^{(2)}\right) .
\end{aligned}
$$

One can show that $\left(1+\tau_{1}^{(1)}(\varepsilon)^{-2}\left(1-\kappa^{2}\right)\left|u_{1}\right|^{2}\right)^{-\frac{\mu}{2}}$ is bounded by const $\cdot\left(1+\left|u_{1}\right|^{2}\right)^{-\frac{\mu}{2}}$ for $\varepsilon$ small enough, where the constant is $\varepsilon$-independent. Because $\mu$ is arbitrary this term decays like an arbitrary power of $\left|u_{1}\right|^{-1}$ when $\left|u_{1}\right| \rightarrow \infty$, in particular faster than $I_{2}$.

Now we take $\mu$ big enough, so that:

$$
I_{1}\left(u_{1}\right) \leqq C_{11}\left(1+\left|u_{1}\right|\right)^{-2-\delta} g^{1}\left(\xi_{2}^{(1)}, \ldots, \xi_{K^{(2)}}^{(2)}\right) .
$$

Then:

$$
\begin{gathered}
\left\|\left(\partial_{\zeta^{\prime}} V_{m n}\left(u_{1}+\tau_{1}^{(1)}(\varepsilon) \xi_{1}^{(1)}\right)\right) g\left(\xi_{1}^{(1)}, \ldots, \xi_{K^{(2)}}^{(2)}\right)\right\|_{L^{2}\left(\mathbb{R}^{3}, d \xi_{1}^{(1)}\right)} \\
\leqq C_{12}\left(1+\left|u_{1}\right|\right)^{-2-\delta} g^{1}\left(\xi_{2}^{(1)}, \ldots, \xi_{K^{(2)}}^{(2)}\right) .
\end{gathered}
$$

As a next step we separate $\xi_{2}^{(1)}$ and follow a similar procedure, choosing:

$$
\begin{aligned}
& B_{1}^{2}=\left\{y:\left|u_{2}+y\right| \leqq \kappa\left|u_{2}\right|\right\}, \\
& B_{2}^{2}=\left\{y:\left|u_{2}+y\right|>\kappa\left|u_{2}\right|\right\},
\end{aligned}
$$

where

$$
u_{2}=\tau(\varepsilon) \zeta+\sum_{k=3}^{K^{(1)}} \tau_{k}^{(1)}(\varepsilon) \xi_{k}^{(1)}+\sum_{k=1}^{K^{(2)}} \tau_{k}^{(2)}(\varepsilon) \xi_{k}^{(2)}
$$

After $\mathrm{N}$ steps we obtain:

$$
\begin{aligned}
& \left\|\partial_{\zeta_{i}} V_{m n}\left(\tau(\varepsilon) \zeta+\sum_{k=1}^{K^{(1)}} \tau_{k}^{(1)}(\varepsilon) \xi_{k}^{(1)}+\sum_{k=1}^{K^{(2)}} \tau_{k}^{(2)}(\varepsilon) \xi_{k}^{(2)}\right) g\left(\xi^{(1)}, \xi^{(2)}\right)\right\|_{L^{2}\left(\mathbb{R}^{3 N}, d \xi\right)} \\
& \leqq C_{13}(1+|\zeta|)^{-2-\delta} .
\end{aligned}
$$

To prove parts b) and c) we compute derivatives explicitly. In part b), for example, we get: 


$$
\begin{aligned}
\partial_{\zeta^{l}} \partial_{\zeta^{j}} P_{\varepsilon}(\zeta) \varphi_{D}(\xi) & \\
= & -\frac{1}{2 \pi i}\left[\int_{\left|z-E_{D}\right|=\gamma}\left(h_{\varepsilon}(\zeta)-z\right)^{-1}\left(\partial_{\zeta^{j}} V_{D, \varepsilon}\right)\left(h_{\varepsilon}(\zeta)-z\right)^{-1}\right. \\
& \times\left(\partial_{\zeta^{i}} V_{D, \varepsilon}\right)\left(h_{\varepsilon}(\zeta)-z\right)^{-1} \varphi_{D}(\xi) d z \\
& -\int_{\left|z-E_{D}\right|=\gamma}\left(h_{\varepsilon}(\zeta)-z\right)^{-1}\left(\partial_{\zeta^{i}} \partial_{\zeta^{j}} V_{D, \varepsilon}\right)\left(h_{\varepsilon}(\zeta)-z\right)^{-1} \varphi_{D}(\xi) d z \\
& +\int_{\left|z-E_{D}\right|=\gamma}\left(h_{\varepsilon}(\zeta)-z\right)^{-1}\left(\partial_{\zeta^{i}} V_{D, \varepsilon}\right)\left(h_{\varepsilon}(\zeta)-z\right)^{-1} \\
& \left.\times\left(\partial_{\zeta^{j}} V_{D, \varepsilon}\right)\left(h_{\varepsilon}(\zeta)-z\right)^{-1} \varphi_{D}(\xi) d z\right] .
\end{aligned}
$$

The middle term can be handled as in part a). To estimate the remaining two terms we write $V_{D, \varepsilon}$ as in (2c). Below we outline the procedure for a generic term $V_{m n}$. We rewrite the integrand as:

$$
\begin{aligned}
\left(h_{\varepsilon}(\zeta)-z\right)^{-1}\left(\partial_{\zeta^{j}} V_{m n}\right) & \cdot\left(1+\left|\tau(\varepsilon) \zeta+\sum_{k=1}^{K^{(1)}} \tau_{k}^{(1)}(\varepsilon) \xi_{k}^{(1)}+\sum_{k=1}^{K^{(2)}} \tau_{k}^{(2)}(\varepsilon) \xi_{k}^{(2)}\right|\right)^{-2-\delta} \\
& \times\left(1+\left|\tau(\varepsilon) \zeta+\sum_{k=1}^{K^{(1)}} \tau_{k}^{(1)}(\varepsilon) \xi_{k}^{(1)}+\sum_{k=1}^{K^{(2)}} \tau_{k}^{(2)}(\varepsilon) \xi_{k}^{(2)}\right|\right)^{2+\delta} \\
& \times\left(h_{\varepsilon}(\zeta)-z\right)^{-1}\left(\partial_{\zeta^{i}} V_{m n}\right)\left(h_{\varepsilon}(\zeta)-z\right)^{-1} \varphi_{D}(\xi)
\end{aligned}
$$

Here $\left(h_{\varepsilon}(\zeta)-z\right)^{-1} \varphi_{D}(\xi) \in L_{\mu}^{2}$ by Lemma $\mathrm{A}$ in the Appendix. If we denote:

$$
\begin{aligned}
g(\xi)= & \left(1+\left|\tau(\varepsilon) \zeta+\sum_{k=1}^{K^{(1)}} \tau_{k}^{(1)}(\varepsilon) \xi_{k}^{(1)}+\sum_{k=1}^{K^{(2)}} \tau_{k}^{(2)}(\varepsilon) \xi_{k}^{(2)}\right|\right)^{2+\delta}\left(h_{\varepsilon}(\zeta)-z\right)^{-1} \\
& \times\left(\partial_{\xi^{i}} V_{m n}\right)\left(h_{\varepsilon}(\zeta)-z\right)^{-1} \varphi_{D}(\xi)
\end{aligned}
$$

then Lemma B shows that $g(\xi)$ is also in $L_{\mu}^{2}$. Thus the proof of b) is reduced to showing that:

$$
\begin{aligned}
& \| \partial_{\zeta^{j}} V_{m n}\left(\tau(\varepsilon) \zeta+\sum_{k=1}^{K^{(1)}} \tau_{k}^{(1)}(\varepsilon) \xi_{k}^{(1)}+\sum_{k=1}^{K^{(2)}} \tau_{k}^{(2)}(\varepsilon) \xi_{k}^{(2)}\right) \\
& \quad \times\left(1+\left|\tau(\varepsilon) \zeta+\sum_{k=1}^{K^{(1)}} \tau_{k}^{(1)}(\varepsilon) \xi_{k}^{(1)}+\sum_{k=1}^{K^{(1)}} \tau_{k}^{(2)}(\varepsilon) \xi_{k}^{(2)}\right|\right)^{-2-\delta} g(\xi) \|_{L^{2}\left(\mathbb{R}^{3 N}, d \xi\right)} \\
& \leqq C_{13}(1+|\zeta|)^{-3-\delta}
\end{aligned}
$$

We use the decay properties of $g(\xi)$ and $\partial_{\zeta^{i}} V_{m n}$ to show conclusion b) exactly the same way as we did for a).

Proof for part c) is similar to b).

Remark. Martinez et al. [12] give a much shorter proof for a similar result (Theorem 2.2). However, we will later refer to our proof several times, hence we present the estimates in detail. 


\section{Corollary 2.}

$$
\left|\left\langle\varphi_{D}, P_{\varepsilon}(\zeta) \varphi_{D}\right\rangle-1\right| \leqq C_{1} \varepsilon^{4}+\theta(\zeta)
$$

where

$$
\theta(\zeta) \leqq C_{2}(1+|\zeta|)^{-1-\delta}
$$

and $\delta$ is as in (3).

Proof. Write $P_{\varepsilon}(\zeta) \varphi_{D}$ as:

$$
\begin{aligned}
P_{\varepsilon}(\zeta) \varphi_{D}= & P_{D} \varphi_{D}+\frac{1}{2 \pi i_{\left|z-E_{D}\right|=\gamma}} \int_{\varepsilon}\left(h_{\varepsilon}(\zeta)-z\right)^{-1}\left[\varepsilon^{4} D(\varepsilon)\right]\left(h_{D}(\zeta)-z\right)^{-1} \varphi_{D} d z \\
& +\frac{1}{2 \pi i_{\left|z-E_{D}\right|=\gamma}} \int_{\varepsilon}\left(h_{\varepsilon}(\zeta)-z\right)^{-1} V_{D, \varepsilon} \cdot\left(h_{D}(\zeta)-z\right)^{-1} \varphi_{D} d z
\end{aligned}
$$

The last term can be estimated as above in part a) of the proof of Lemma 1, yielding:

$$
\left.\| \frac{1}{2 \pi i_{\left|z-E_{D}\right|=\gamma}} \int_{\varepsilon}(\zeta)-z\right)^{-1} V_{D, \varepsilon}\left(h_{D}(\zeta)-z\right)^{-1} \varphi_{D} d z \|_{L^{2}\left(\mathbb{R}^{3 N}, d \xi\right)} \leqq C_{3}(1+|\zeta|)^{-1-\delta} .
$$

In the middle term we note that $D(\varepsilon)$ is relatively bounded with respect to $h_{D}$, so:

$$
\begin{aligned}
& \left\|\frac{\varepsilon^{4}}{2 \pi i_{\left|z-E_{D}\right|=\gamma}}\left(h_{\varepsilon}-z\right)^{-1} D(\varepsilon)\left(h_{D}-z\right)^{-1} \varphi_{D} d z\right\| \\
& \quad \leqq \frac{\varepsilon^{4}}{2 \pi} \int_{\left|z-E_{D}\right|=\gamma}\left\|\left(h_{\varepsilon}-z\right)^{-1}\right\|_{\mathrm{op}}\left\|D(\varepsilon)\left(h_{D}-z\right)^{-1} \varphi_{D}\right\| d z \\
& \quad \leqq C_{4} \varepsilon^{4} \int_{\left|z-E_{D}\right|=\gamma}\left(a\left\|h_{D}\left(h_{D}-z\right)^{-1} \varphi_{D}\right\|+b\left\|\left(h_{D}-z\right)^{-1} \varphi_{D}\right\|\right) d z \\
& \quad \leqq C_{5} \varepsilon^{4} .
\end{aligned}
$$

Now we denote:

$$
\tilde{E}_{\varepsilon}(\zeta)=E_{\varepsilon}(\zeta)-E_{D}=\frac{\left\langle P_{\varepsilon}(\zeta) \varphi_{D}(\xi),\left(\varepsilon^{4} D(\varepsilon)+V_{D, \varepsilon}\right) \varphi_{D}(\xi)\right\rangle}{\left\langle\varphi_{D}(\xi), P_{\varepsilon}(\zeta) \varphi_{D}(\xi)\right\rangle}
$$

Using Lemma 1 and the above corollary we can bound the corrections to the energy level.

\section{Lemma 3.}

$$
\left|\tilde{E}_{\varepsilon}^{(\alpha)}(\zeta)\right| \leqq C(1+|\zeta|)^{-1-\alpha-\delta}
$$

for $\alpha=1,2,3$, where the superscript $(\alpha)$ means the partial derivatives of $\tilde{E}_{\varepsilon}$ of order $\alpha$ with respect to the components of $\zeta$.

Proof. We use estimates:

$$
\left\|V_{D, \varepsilon}^{(\alpha)} \varphi_{D}\right\|_{L^{2}(d \xi)} \leqq C_{\alpha}(1+|\zeta|)^{-1-\alpha-\delta}
$$


for $\alpha=1,2,3, \varepsilon$ small enough, which follow from our assumptions on $V_{m n}$. The proof of this is similar to the estimates in the proof of Lemma 1, part a).

Also, as mentioned above, $D(\varepsilon)$ is relatively bounded with respect to $h_{D}$.

To prove the lemma we compute derivatives of $\tilde{E}_{\varepsilon}(\zeta)$ explicitly. For $\alpha=1$, for instance, we get:

$$
\begin{aligned}
\partial_{\zeta^{i}} \tilde{E}_{\varepsilon}(\zeta)= & \frac{\left\langle\partial_{\zeta^{i}} P_{\varepsilon}(\zeta) \varphi_{D}(\xi),\left(\varepsilon^{4} D(\varepsilon)+V_{D, \varepsilon}\right) \varphi_{D}(\xi)\right\rangle+\left\langle P_{\varepsilon}(\zeta) \varphi_{D}(\xi),\left(\partial_{\zeta^{i}} V_{D, \varepsilon}\right) \varphi_{D}(\xi)\right\rangle}{\left\langle\varphi_{D}(\xi), P_{\varepsilon}(\zeta) \varphi_{D}(\xi)\right\rangle} \\
& -\frac{\left\langle P_{\varepsilon}(\zeta) \varphi_{D}(\xi),\left(\varepsilon^{4} D(\varepsilon)+V_{D, \varepsilon}\right) \varphi_{D}(\xi)\right\rangle\left\langle\varphi_{D}(\xi), \partial_{\zeta^{i}} P_{\varepsilon}(\zeta) \varphi_{D}(\xi)\right\rangle}{\left(\left\langle\varphi_{D}(\xi), P_{\varepsilon}(\zeta) \varphi_{D}(\xi)\right\rangle\right)^{2}} .
\end{aligned}
$$

Then we use the Schwarz lemma, Lemma 1, Corollary 2 and the above estimates on $\left\|V_{D, \varepsilon}^{(\alpha)} \varphi_{D}\right\|_{L^{2}(d \dot{\xi})}$.

Remark. By a similar argument one can show:

$$
\left|E_{\varepsilon}(\zeta)-E_{D}\right| \leqq C \varepsilon^{4}+C^{\prime}(1+|\zeta|)^{-1-\delta}
$$

\section{The Born-Oppenheimer Approximation}

Our goal, as mentioned in the introduction, is to construct the approximate solution to the time-dependent Schrödinger equation, which is asymptotic to the exact one in the leading order. We begin by stating our main result.

First fix $3 \times 3$ matrices $A_{+}, B_{+}$and vectors $a_{+}, \eta_{+} \in \mathbb{R}^{3}, \eta_{+} \neq 0$. By Lemma 3 and [5] there exists a solution $a_{\varepsilon}(t), \eta_{\varepsilon}(t), A_{\varepsilon}(t), B_{\varepsilon}(t)$ to the system (9) satisfying the asymptotic conditions (10). Then we have:

Theorem. Let $H$ be defined by Eq. (1),(2). Assume the potentials satisfy (3). Also assume the conditions on $E_{D}$ and $E_{\varepsilon}(\zeta)$ mentioned in the previous section are satisfied. Let $a_{\varepsilon}(t), \eta_{\varepsilon}(t), A_{\varepsilon}(t), B_{\varepsilon}(t)$ be as above. Then the function:

$$
\begin{aligned}
\psi_{\varepsilon}(\xi, \zeta, t)= & e^{\frac{i S(t)}{\varepsilon^{2}}} \varphi\left(A_{\varepsilon}(t), B_{\varepsilon}(t), \varepsilon^{2}, a_{\varepsilon}(t), \eta_{\varepsilon}(t), \zeta\right) \\
& \times\left[\varphi_{\varepsilon}(\xi, \zeta)+i \varepsilon^{2} r(\zeta) \eta_{\varepsilon}(t) \cdot \nabla_{\zeta} \varphi_{\varepsilon}(\xi, \zeta)\right],
\end{aligned}
$$

where $r(\zeta)$ is the reduced resolvent of $h_{\varepsilon}(\zeta)$ at $E_{\varepsilon}(\zeta)$, is the 0 -th order asymptotic expansion of the solution to equation:

$$
i \varepsilon^{2} \frac{\partial \Psi(\xi, \zeta, t)}{\partial t}=\left[-\frac{\varepsilon^{4}}{2} \Delta_{\zeta}+h_{\varepsilon}(\zeta)\right] \Psi(\xi, \zeta, t),
$$

i.e. the following holds:

$$
\left\|e^{-\frac{i t H}{\varepsilon^{2}}} \psi_{\varepsilon}(\xi, \zeta, 0)-\psi_{\varepsilon}(\xi, \zeta, t)\right\| \leqq C \varepsilon
$$

for all $t \in[0, \infty)$.

Our main tool will be the following simple lemma [7]:

Lemma 4. Let $\Psi(t)$ be a differentiable vector-valued function, whose values belong to the domain of $H$ for all $t \in[0, \infty)$. If $\Psi$ satisfies: 


$$
i \varepsilon^{2} \dot{\Psi}=H \Psi+\lambda(t)
$$

where

$$
\|\lambda(t)\| \leqq F(t) \varepsilon^{3}
$$

for some $F \in L^{1}(\mathbb{R}, d t)$, then:

$$
\left\|e^{\frac{i t H}{\varepsilon^{2}}} \Psi(0)-\Psi(t)\right\| \leqq C \varepsilon
$$

where $C=\int_{0}^{\infty} F(t) d t$.

To prove our theorem we first formally construct the candidate for the approximate solution. Then we substitute it to Eq. (18) and calculate the error term $\lambda(t)$. Finally we show that the error term satisfies (19) and the application of Lemma 4 finishes the proof.

The details of these formal calculations have been shown in Sect. 3 of [7]. Here we only briefly describe the method used and state the results.

We use the so-called multiple scales technique to separate the adiabatic and semiclassical aspects of the problem. It consists in seeking the solution $\Phi(\xi, x, y, t)$ of the higher dimensional problem defined by a formal change of variables from $(\xi, \zeta, t)$ to $(\xi, x, y, t)$, where $x=\zeta, y=\frac{\zeta-a_{\varepsilon}(t)}{\varepsilon}$, in the equation:

$$
i \varepsilon^{2} \frac{\partial \Psi(\xi, \zeta, t)}{\partial t}=\left[-\frac{\varepsilon^{4}}{2} \Delta_{\zeta}+h_{\varepsilon}(\zeta)\right] \Psi(\xi, \zeta, t)
$$

The result is:

$$
\begin{aligned}
i \varepsilon^{2} \frac{\partial \Phi}{\partial t}= & {\left[-\frac{\varepsilon^{4}}{2} \Delta_{x}-\varepsilon^{3} \nabla_{x} \cdot \nabla_{y}-\frac{\varepsilon^{2}}{2} \Delta_{y}\right.} \\
& \left.+i \varepsilon \eta_{\varepsilon}(t) \cdot \nabla_{y}+E_{\varepsilon}\left(a_{\varepsilon}(t)+\varepsilon y\right)+h_{\varepsilon}(x)-E_{\varepsilon}(x)\right] \Phi
\end{aligned}
$$

We formally solve (22) assuming the solution in the form of power series in $\varepsilon$ and collecting terms of the same order in $\varepsilon$. This generates a formal solution and finally, substituting back $x=\zeta, y=\frac{\zeta-a_{\varepsilon}(t)}{\varepsilon}$ we recover $\Psi(\xi, \zeta, t)-$ the formal solution to (21). The explicit formulae can be found in [7].

For our purposes it is important to note that the only terms contributing to the 0 -th order approximation are:

$$
\begin{gathered}
\Psi_{0, \varepsilon}=\varepsilon^{-\frac{3}{2}} \varphi\left(A_{\varepsilon}(t), B_{\varepsilon}(t), 1,0,0, y\right) \varphi_{\varepsilon}(\xi, x), \\
\Psi_{2, \varepsilon}^{\perp}=\operatorname{ir}(x) \eta_{\varepsilon}(t) \cdot \nabla_{x} \Psi_{0, \varepsilon},
\end{gathered}
$$

where $r(x)$ is the reduced resolvent of $h_{\varepsilon}(x)$ at $E_{\varepsilon}(x)$ :

$$
r(x)=\left[h_{\varepsilon}(x)-E_{\varepsilon}(x)\right]^{-1}\left(1-P_{\varepsilon}(x)\right)
$$

and $P_{\varepsilon}(x)$ is the spectral projector associated with the eigenvalue $E_{\varepsilon}(x)$. The function:

$$
\Phi_{\varepsilon}(\xi, x, y, t)=e^{i \frac{S(t)}{\varepsilon^{2}}} e^{i \frac{\eta_{\varepsilon}(t) \cdot y}{\varepsilon}}\left(\Psi_{0, \varepsilon}+\varepsilon^{2} \Psi_{2, \varepsilon}^{\perp}\right)
$$

will serve as a candidate for our approximate solution. Note that upon returning to our original variables $\xi, \zeta, t$ the function (24) becomes (15). 


\section{Proof of the Theorem}

We remain in the multiple scales framework and calculate the error terms in Eq. (18) using the function (24). The result is:

$$
\begin{aligned}
\lambda(t)= & e^{\frac{i S(t)}{\varepsilon^{2}}} e^{\frac{i \eta_{\varepsilon}(t) \cdot y}{\varepsilon}}\left\{\varepsilon^{3} \nabla_{y} f_{0, \varepsilon}(y) \cdot \nabla_{x} \varphi_{\varepsilon}(\xi, x)\right. \\
& +\varepsilon^{4}\left[f_{0, \varepsilon}(y) r(x) E_{\varepsilon}^{\prime}\left(a_{\varepsilon}(t)\right) \cdot \nabla_{x} \varphi_{\varepsilon}(\xi, x)+\frac{1}{2} f_{0, \varepsilon}(y) \Delta_{x} \varphi_{\varepsilon}(\xi, x)\right. \\
& \left.-f_{0, \varepsilon}(y) \eta_{\varepsilon}(t) \cdot \nabla_{x} \varphi_{1, \varepsilon}(\xi, x)\right] \\
& +\varepsilon^{5} i \nabla_{y} f_{0, \varepsilon}(y) \cdot \nabla_{x} \varphi_{1, \varepsilon}(\xi, x) \\
& +\varepsilon^{6} \frac{i}{2} f_{0, \varepsilon}(y) \Delta_{x} \varphi_{1, \varepsilon}(\xi, x) \\
& -\left[E_{\varepsilon}\left(a_{\varepsilon}(t)+\varepsilon y\right)-E_{\varepsilon}\left(a_{\varepsilon}(t)\right)-\varepsilon y E_{\varepsilon}^{\prime}\left(a_{\varepsilon}(t)\right)-\frac{\varepsilon^{2} y^{2}}{2} E_{\varepsilon}^{\prime \prime}\left(a_{\varepsilon}(t)\right)\right] \\
& \left.\times\left[f_{0, \varepsilon}(y) \varphi_{\varepsilon}(\xi, x)+i \varepsilon^{2} f_{0, \varepsilon}(y) \varphi_{1, \varepsilon}(\xi, x)\right]\right\},
\end{aligned}
$$

where:

$$
\begin{aligned}
f_{0, \varepsilon}(y) & =\varepsilon^{-\frac{3}{2}} \varphi\left(A_{\varepsilon}(t), B_{\varepsilon}(t), 1,0,0, y\right), \\
\varphi_{1, \varepsilon}(\xi, x) & =r(x) \eta_{\varepsilon}(t) \cdot \nabla_{x} \varphi_{\varepsilon}(\xi, x) .
\end{aligned}
$$

We begin analysis with the first four terms. They are basically of the form $f_{0, \varepsilon}(y)$ (or $\nabla_{y} f_{0, \varepsilon}(y)$ ) multiplied by $\nabla_{x} \varphi_{\varepsilon}$ (or $\nabla_{x} \varphi_{1, \varepsilon}$ ). Note that the first factor is concentrated near $\zeta=a_{\varepsilon}(t)$ while the second is near $\zeta=0$. Using their decay properties we can prove desired estimates.

Lemma 5. Let $\omega(x)$ be a function $\mathbb{R}^{3} \rightarrow \mathbb{R}$ satisfying $|\omega(x)| \leqq(1+|x|)^{-1-\delta}$, and let $f_{0, \varepsilon}(y)$ be as defined above. Then:

$$
\left\|f_{0, \varepsilon}\left(\frac{\zeta-a_{\varepsilon}(t)}{\varepsilon}\right) \omega(\zeta)\right\|_{L^{2}\left(\mathbb{R}^{3}, d \zeta\right)} \leqq F(t)
$$

for some $F \in L^{1}(\mathbb{R}, d t)$.

Proof. For $0<\mu<1$ we define the sets:

$$
\begin{aligned}
& B_{1}=\left\{\zeta:\left|\zeta-a_{\varepsilon}(t)\right| \leqq \mu\left|a_{\varepsilon}(t)\right|\right\}, \\
& B_{2}=\left\{\zeta:\left|\zeta-a_{\varepsilon}(t)\right|>\mu\left|a_{\varepsilon}(t)\right|\right\},
\end{aligned}
$$

and let $\chi_{1}, \chi_{2}$ be the corresponding characteristic functions. Then:

$$
\left\|f_{0, \varepsilon} \omega\right\|_{2}=\left\|\chi_{1} f_{0, \varepsilon} \omega\right\|_{2}+\left\|\chi_{2} f_{0, \varepsilon} \omega\right\|_{2}=I_{1}(t)+I_{2}(t) .
$$

By the Hölder inequality:

$$
I_{1}(t)=\left\|\chi_{1}(\zeta) \omega(\zeta) f_{0, \varepsilon}\left(\frac{\zeta-a_{\varepsilon}(t)}{\varepsilon}\right)\right\|_{2} \leqq\left\|\chi_{1}(\zeta) \omega(\zeta)\right\|_{\infty}\left\|f_{0, \varepsilon}\left(\frac{\zeta-a_{\varepsilon}(t)}{\varepsilon}\right)\right\|_{2}
$$


The second factor equals 1 and from the definition of the set $B_{1}$ we see that the first factor is bounded by $C_{1}\left(1+(1-\mu)\left|a_{\varepsilon}(t)\right|\right)^{-1-\delta}$.

For $I_{2}$ we have:

$$
\begin{aligned}
I_{2}(t)= & || \chi_{2}(\zeta) e^{-\frac{1}{4 \varepsilon^{2}}\left|A_{\varepsilon}(t)^{-1}\left(\zeta-a_{\varepsilon}(t)\right)\right|^{2}}\left\|_{\infty}\right\| \omega(\zeta) \pi^{-\frac{3}{4}} \varepsilon^{-\frac{3}{2}} \\
& \times\left|\operatorname{det} A_{\varepsilon}(t)\right|^{-\frac{1}{2}} e^{-\frac{1}{4 \varepsilon^{2}}\left|A_{\varepsilon}(t)^{-1}\left(\zeta-a_{\varepsilon}(t)\right)\right|^{2}} \|_{2} \\
\leqq & \left\|\chi_{2}(\zeta) e^{-\frac{1}{4 \varepsilon^{2}}\left|A_{\varepsilon}(t)^{-1}\left(\zeta-a_{\varepsilon}(t)\right)\right|^{2}}\right\|_{\infty}\|\omega\|_{q} \| \pi^{-\frac{3}{4}} \varepsilon^{-\frac{3}{2}} \\
& \times\left|\operatorname{det} A_{\varepsilon}(t)\right|^{-\frac{1}{2}} e^{-\frac{1}{4 \varepsilon^{2}}\left|A_{\varepsilon}(t)^{-1}\left(\zeta-a_{\varepsilon}(t)\right)\right|^{2}} \|_{p},
\end{aligned}
$$

where $\frac{1}{p}+\frac{1}{q}=\frac{1}{2}$.

If we take $\frac{3}{1+\delta}<q<\frac{3}{1+\frac{\delta}{2}}$, then $\|\omega\|_{q}=C_{2}<\infty$ and $-\frac{3}{q}<-\left(1+\frac{\delta}{2}\right)$. The last factor equals:

$$
\begin{aligned}
& \left\|\pi^{-\frac{3}{4}} \varepsilon^{-\frac{3}{2}}\left|\operatorname{det} A_{\varepsilon}(t)\right|^{-\frac{1}{2}} e^{-\frac{1}{4 \varepsilon^{2}}\left|A_{\varepsilon}(t)^{-1}\left(\zeta-a_{\varepsilon}(t)\right)\right|^{2}}\right\|_{p} \\
& =C_{3} \varepsilon^{-3\left(\frac{1}{2}-\frac{1}{p}\right)}\left|\operatorname{det} A_{\varepsilon}(t)\right|^{-\left(\frac{1}{2}-\frac{1}{p}\right)} \\
& =C_{3} \varepsilon^{-\frac{3}{q}}\left|\operatorname{det} A_{\varepsilon}(t)\right|^{-\frac{1}{q}} .
\end{aligned}
$$

The first factor is bounded by:

$$
e^{-\frac{1}{4 \varepsilon^{2}}\left\|A_{\varepsilon}(t)\right\|^{-2} \mu^{2}\left|a_{\varepsilon}(t)\right|^{2}}
$$

which, because of the asymptotics of $A_{\varepsilon}(t)$ and $a_{\varepsilon}(t)$, is bounded by $e^{-C_{4} \varepsilon^{-2}}$ for all $t$. Thus we have the following bound on $I_{2}(t)$ :

$$
I_{2}(t) \leqq C_{5} e^{-C_{4} \varepsilon^{-2}} \varepsilon^{-\frac{3}{q}}\left|\operatorname{det} A_{\varepsilon}(t)\right|^{-\frac{1}{q}} .
$$

This bound decays to 0 as $\varepsilon \rightarrow 0$, thus we can find $C_{6}$ such that $I_{2}(t) \leqq$ $C_{6}\left|\operatorname{det} A_{\varepsilon}(t)\right|^{-\frac{1}{q}}$. Now, asymptotic behavior of $A_{\varepsilon}(t)$ and our choice of $q$ guarantee the existence of a $L^{1}$-function $F_{2}(t)$ such that:

$$
I_{2}(t) \leqq C_{6} F_{2}(t) .
$$

Combining the two bounds (for $I_{1}$ and $I_{2}$ ) together we get the lemma.

Remark. A similar conclusion holds if we multiply $f_{0, \varepsilon}$ by any polynomial in $y$.

We will further need this bound for $f_{0, \varepsilon}$ as well as for $\nabla_{y} f_{0, \varepsilon}$, thus we sketch the proof for the latter case below. We keep notation of the proof of Lemma 5.

We calculate $\nabla_{y} f_{0, \varepsilon}$ explicitly:

$$
\nabla_{y} f_{0, \varepsilon}(y)=-C_{7} \varepsilon^{-\frac{3}{2}}\left(\operatorname{det} A_{\varepsilon}(t)\right)^{-\frac{1}{2}} B_{\varepsilon}(t) A_{\varepsilon}(t)^{-1} y e^{-\frac{1}{2}\left\langle y, B_{\varepsilon}(t) A_{\varepsilon}(t)^{-1} y\right\rangle} .
$$

Then:

$$
\begin{gathered}
\left\|\nabla_{y} f_{0, \varepsilon}\left(\frac{\zeta-a_{\varepsilon}(t)}{\varepsilon}\right)\right\|_{2}=C_{7} \varepsilon^{-\frac{3}{2}}\left(\operatorname{det} A_{\varepsilon}(t)\right)^{-\frac{1}{2}} \\
\left(\int\left|B_{\varepsilon}(t) A_{\varepsilon}(t)^{-1} \frac{\zeta-a_{\varepsilon}(t)}{\varepsilon}\right|^{2} e^{-\frac{1}{\varepsilon^{2}}\left\langle\zeta-a_{\varepsilon}(t), A_{\varepsilon}(t)^{*-1} A_{\varepsilon}(t)^{-1}\left(\zeta-a_{\varepsilon}(t)\right)\right\rangle} d^{3} \zeta\right)^{\frac{1}{2}}
\end{gathered}
$$


After change of variables to $u=A_{\varepsilon}(t)^{-1} \frac{\zeta-a_{\varepsilon}(t)}{\varepsilon}$, we see that (27) is bounded by $C_{8}\left\|B_{\varepsilon}(t)\right\|_{\text {op. }}$. Since the operator norm of matrix $B_{\varepsilon}(t)$ tends to a constant as $t \rightarrow \infty$ we get for $I_{1}$ a bound similar to that in the proof of Lemma 5. The estimate for $I_{2}$ is essentially the same as in Lemma 5.

Lemma 6. Assume $h_{\varepsilon}(x), E_{\varepsilon}(x)$ are as described in the introduction. Let $\beta>0$ be such that $h_{D}$ has no eigenvalues in the closed ball $\overline{B\left(E_{D}, \beta\right)}$ other than $E_{D}$. Let $|x|$ be big enough so $E_{\varepsilon}(x) \in B\left(E_{D}, \frac{\beta}{2}\right)$ and $h_{\varepsilon}(x)$ has no other eigenvalues in $B\left(E_{D}, \beta\right)$. Then the reduced resolvent $\left(h_{\varepsilon}(x)-E_{\varepsilon}(x)\right)^{-1}\left(1-P_{\varepsilon}(x)\right)$ and its first and second derivatives are bounded uniformly in $x$.

Proof. Look at $\left(h_{\varepsilon}(x)-z\right)^{-1}\left(1-P_{\varepsilon}(x)\right)$. This is the analytic part of the Laurent series of $\left(h_{\varepsilon}(x)-z\right)^{-1}$ around $E_{\varepsilon}(x)$, thus it has a removable singularity at $E_{\varepsilon}(x)$. By the assumption on the eigenvalues it can be continued analytically to the entire ball $B\left(E_{D}, \beta\right)$.

If we restrict it to $B\left(E_{D}, \frac{\beta}{2}\right)$, then by maximum modulus theorem it assumes its maximal value on the boundary $\partial B\left(E_{D}, \frac{\beta}{2}\right)$ i.e.:

$$
\begin{aligned}
\left\|\left(h_{\varepsilon}(x)-E_{\varepsilon}(x)\right)^{-1}\left(1-P_{\varepsilon}(x)\right)\right\|_{\mathrm{op}} & \leqq \sup _{\left|z-E_{D}\right|=\frac{\beta}{2}}\left\|\left(h_{\varepsilon}(x)-z\right)^{-1}\left(1-P_{\varepsilon}(x)\right)\right\|_{\mathrm{op}} \\
& \leqq \sup _{\left|z-E_{D}\right|=\frac{\beta}{2}}\left\|\left(h_{\varepsilon}(x)-z\right)^{-1}\right\|_{\mathrm{op}} \leqq \frac{2}{\beta}
\end{aligned}
$$

uniformly in $x$. This proves the first conclusion of the lemma.

To show boundedness of the derivative we write the reduced resolvent as:

$$
\left(h_{\varepsilon}(x)-z\right)^{-1}\left(1-P_{\varepsilon}(x)\right)=\left(1-P_{\varepsilon}(x)\right)\left(h_{\varepsilon}(x)-z\right)^{-1}\left(1-P_{\varepsilon}(x)\right)
$$

and compute the derivative:

$$
\begin{aligned}
& \partial_{x^{i}}\left(\left(h_{\varepsilon}(x)-z\right)^{-1}\left(1-P_{\varepsilon}(x)\right)\right)=-\left(\partial_{x^{i}} P_{\varepsilon}(x)\right)\left(h_{\varepsilon}(x)-z\right)^{-1}\left(1-P_{\varepsilon}(x)\right) \\
& \quad-\left(1-P_{\varepsilon}(x)\right)\left(h_{\varepsilon}(x)-z\right)^{-1}\left(\partial_{x^{l}} V_{D, \varepsilon}(x)\right)\left(h_{\varepsilon}(x)-z\right)^{-1}\left(1-P_{\varepsilon}(x)\right) \\
& \quad-\left(h_{\varepsilon}(x)-z\right)^{-1}\left(1-P_{\varepsilon}(x)\right)\left(\partial_{x^{i}} P_{\varepsilon}(x)\right)
\end{aligned}
$$

Our assumptions on $V_{D, \varepsilon}$ guarantee that the operator norm of $\partial_{x^{i}} V_{D, \varepsilon}$ is a constant.

To analyze $\partial_{x^{l}} P_{\varepsilon}(x)$ we write:

$$
\begin{aligned}
\left\|\partial_{x^{i}} P_{\varepsilon}(x)\right\| & \leqq \frac{1}{2 \pi} \int_{\left|z-E_{D}\right|=\frac{\beta}{2}}\left\|\left(h_{\varepsilon}(x)-z\right)^{-1}\right\|^{2}\left\|\partial_{x^{i}} V_{D, \varepsilon}(x)\right\| d z \\
& \leqq C \sup _{\left|z-E_{D}\right|=\frac{\beta}{2}}\left\|\left(h_{\varepsilon}(x)-z\right)^{-1}\right\|^{2}\left\|\partial_{x^{i}} V_{D, \varepsilon}(x)\right\| \\
& \leqq C^{\prime}
\end{aligned}
$$

uniformly in $x$.

Since the reduced resolvent has just been shown to be uniformly bounded, the conclusion holds for the first derivative of the reduced resolvent.

For the second derivative we prove the estimate in a similar way. We write (28) as: 


$$
\begin{aligned}
& \partial_{x^{i}}\left(\left(h_{\varepsilon}(x)-z\right)^{-1}\left(1-P_{\varepsilon}(x)\right)\right)=-\left(\partial_{x^{i}} P_{\varepsilon}(x)\right)\left(1-P_{\varepsilon}(x)\right)\left(h_{\varepsilon}(x)-z\right)^{-1}\left(1-P_{\varepsilon}(x)\right) \\
& \quad-\left(1-P_{\varepsilon}(x)\right)\left(h_{\varepsilon}(x)-z\right)^{-1}\left(1-P_{\varepsilon}(x)\right)\left(\partial_{x^{i}} V_{D, \varepsilon}(x)\right) \\
& \quad \times\left(1-P_{\varepsilon}(x)\right)\left(h_{\varepsilon}(x)-z\right)^{-1}\left(1-P_{\varepsilon}(x)\right) \\
& \quad-\left(1-P_{\varepsilon}(x)\right)\left(h_{\varepsilon}(x)-z\right)^{-1}\left(1-P_{\varepsilon}(x)\right)\left(\partial_{x^{i}} P_{\varepsilon}(x)\right)
\end{aligned}
$$

and compute the second derivative of $P_{\varepsilon}(x)$ explicitly. It consists of terms containing products of the reduced resolvent, derivatives of $V_{D, \varepsilon}$ and derivatives of $P_{\varepsilon}(x)$ up to the second order. All of these factors, except $\partial_{x^{i}} \partial_{x^{j}} P_{\varepsilon}(x)$, have been shown to be bounded uniformly in $x$. To conclude the same for $\partial_{x^{i}} \partial_{x^{j}} P_{\varepsilon}(x)$ we proceed exactly the same way as we did to show boundedness of the first derivative of $P_{\varepsilon}(x)$. Finally we collect all these bounds to prove the last conclusion of the lemma.

Now we have tools to estimate all but the last term in (25). We first look at the $x$-dependent factors. Recall that the normalized eigenfunction $\varphi_{\varepsilon}(\xi, x)$ of $h_{\varepsilon}(x)$ is of the form:

$$
\varphi_{\varepsilon}(\xi, x)=\frac{P_{\varepsilon}(x) \varphi_{D}(\xi)}{\left\langle\varphi_{D}(\xi), P_{\varepsilon}(x) \varphi_{D}(\xi)\right\rangle} .
$$

By explicit calculations and using Lemma 1 and Corollary 2 we conclude that:

$$
\left\|\partial_{x^{i}} \varphi_{\varepsilon}(\xi, x)\right\| \leqq C(1+|x|)^{-2-\delta} .
$$

We can also use Lemma 1 to show similar bounds on the second and third derivatives of $\varphi_{\varepsilon}(\xi, x)$.

Combining (29) (and corresponding bounds for higher derivatives of $\varphi_{\varepsilon}(\xi, x)$ ) with Lemma 6 we see that the $x$-dependent terms in (25) are the products of $\left|\eta_{\varepsilon}(t)\right|$ and functions satisfying the hypothesis of Lemma 5 . Here we return to our original variables by putting $x=\zeta, y=\frac{\zeta-a(t)}{\varepsilon}$. By Lemma 5 and asymptotics of $\eta_{\varepsilon}(t)$ we see that all considered terms are bounded in norm by $C \cdot F(t)$ with $F \in L^{1}(\mathbb{R}, d t)$.

Note: In the above considerations we temporarily disregarded the factor $E_{\varepsilon}^{\prime}(a(t))$ appearing in one of the terms. We need to show that this factor remains bounded as $t \rightarrow \infty$. Lemma 3 says that it behaves even more nicely - it decays to 0 .

To estimate the last term in (25) we use (14). Then, with an obvious abuse of notation, this term becomes:

$$
\begin{aligned}
{\left[\tilde{E}_{\varepsilon}(a(t)\right.} & \left.\left.+\varepsilon y)-\tilde{E}_{\varepsilon}(a(t))-\varepsilon y \cdot \tilde{E}_{\varepsilon}^{\prime} a(t)\right)-\frac{\varepsilon^{2} y^{2}}{2} \tilde{E}_{\varepsilon}^{\prime \prime}(a(t))\right] \\
& \times\left[f_{0, \varepsilon}(y) \varphi_{\varepsilon}(\xi, x)+i \varepsilon^{2} f_{0, \varepsilon}(y) \varphi_{1, \varepsilon}(\xi, x)\right] .
\end{aligned}
$$

We note that $\left\|\varphi_{\varepsilon}(\xi, x)+i \varepsilon^{2} \varphi_{1, \varepsilon}(\xi, x)\right\|_{L^{2}(d \xi)}$ is a bounded function of $x$. Also by Lemma 3 we know that $\tilde{E}_{\varepsilon}$ satisfies the hypothesis of Lemma 3.2 of [5]. This shows that the $L^{2}(d \zeta)$-norm of $(30)$ is bounded by $F(t) \varepsilon^{3}$ for some $F \in L^{1}(\mathbb{R}, d t)$. Application of Lemma 4 concludes the proof.

\section{Appendix}

Lemma A. Let $V$ be a potential satisfying $(3 a, b), H_{0}=-\Delta$. Then for $z \notin$ $\sigma\left(H_{0}+V\right)$ the resolvent $\left(H_{0}+V-z\right)^{-1}$ is a bounded operator from $L_{\mu}^{2}$ to $L_{\mu}^{2}$ for arbitrary $\mu$. 
Proof. The proof for $0<\mu \leqq 1$ and $V=0$ can be found in [14], p. 170. Here we outline the general inductive argument. This will also make it clear how one can generalize this result to obtain Lemma B below.

if

We want to show:

$$
\left(H_{0}+V-z\right)^{-1} \text { is bounded } L_{n}^{2} \rightarrow L_{n}^{2}
$$

and $\partial_{i}\left(H_{0}+V-z\right)^{-1}$ is bounded $L_{n}^{2} \rightarrow L_{n}^{2}$,

then

$$
\begin{gathered}
\left(H_{0}+V-z\right)^{-1} \text { and } \partial_{i}\left(H_{0}+V-z\right)^{-1} \\
\text { are bounded } L_{n+\delta}^{2} \rightarrow L_{n+\delta}^{2} \text { for } 0<\delta \leqq 1 .
\end{gathered}
$$

First we show the induction hypotheses for $n=0$. To simplify the notation we write $\mathrm{R}$ for $\left(H_{0}+V-z\right)^{-1}$ and put $\rho=\left(1+|x|^{2}\right)^{\frac{1}{2}}$.

a) $\left(H_{0}+V-z\right)^{-1}$.

We start with formal calculations:

$$
\left[R, \rho^{\delta}\right]=-R\left[H_{0}, \rho^{\delta}\right] R=\sum_{i}\left\{R \partial_{i}\right\}\left(\partial_{i} \rho^{\delta}\right) R+R\left(\partial_{i} \rho^{\delta}\right)\left\{\partial_{i} R\right\}
$$

Applied to Schwarz functions the computations are legitimate. Moreover $R, \partial_{i} R$, and multiplication by $\partial_{i} \rho^{\delta}$ are bounded on $L^{2}$. Thus:

$$
\left\|\left[R, \rho^{\delta}\right] \Psi\right\| \leqq \mathrm{const}\|\Psi\|
$$

for any $\Psi \in L^{2}$. Then we conclude:

$$
\begin{aligned}
\|R \Psi\|_{\delta} & :=\left\|\rho^{\delta} R \Psi\right\| \\
& \leqq\left\|R \rho^{\delta} \Psi\right\|+\left\|\left[R, \rho^{\delta}\right] \Psi\right\| \leqq \mathrm{const}\|\Psi\|_{\delta},
\end{aligned}
$$

i.e. $\left(H_{0}+V-z\right)^{-1}$ is bounded $L_{\delta}^{2} \rightarrow L_{\delta}^{2}$ for $0<\delta \leqq 1$.

b) $\partial_{i}\left(H_{0}+V-z\right)^{-1}$.

Similarly:

$$
\left\|\rho^{\delta} \partial_{i} R \Psi\right\| \leqq\left\|\partial_{i} R \rho^{\delta} \Psi\right\|+\left\|\left[\rho^{\delta}, \partial_{i} R\right] \psi\right\| .
$$

The first term on the r.h.s. is bounded by const $\left\|\rho^{\delta} \Psi\right\|$ since $\partial_{i} R$ is bounded on $L^{2}$. We expand the commutator in the second term as:

$$
\begin{aligned}
{\left[\rho^{\delta}, \partial_{i} R\right] } & =\rho^{\delta} \partial_{i} R-\partial_{i} R \rho^{\delta} \\
& =R\left[H_{0}, \rho^{\delta}\right] \partial_{i} R-R\left(\partial_{i} R\right) R\left[H_{0}, \rho^{\delta}\right] R-R\left(\partial_{i} \rho^{\delta}\right) .
\end{aligned}
$$

All terms are bounded on $L^{2}$, therefore:

$$
\left\|\left[\rho^{\delta}, \partial_{i} R\right] \Psi\right\| \leqq \mathrm{const}\|\Psi\| \leqq \mathrm{const}\|\Psi\|_{\delta} .
$$

Thus the second hypothesis in (A1) holds for $n=0$.

c) We also estimate $\left(H_{0}+V-z\right)^{-1} \partial_{i}$.

Note that $\partial_{i} R$ and $R \partial_{i}$ differ only by $-R\left(\partial_{i} V\right) R$, i.e.

$$
\left\|R \partial_{i} \Psi\right\|_{\delta} \leqq\left\|\partial_{i} R \Psi\right\|_{\delta}+\left\|R\left(\partial_{i} V\right) R \Psi\right\|_{\delta} .
$$


The first term of the r.h.s. is bounded by const $\|\Psi\|_{\delta}$. For the second one we get:

$$
\left\|\rho^{\delta} R\left(\partial_{i} V\right) R \Psi\right\| \leqq\left\|R\left(\partial_{i} V\right) R \rho^{\delta} \Psi\right\|+\left\|R\left(\partial_{i} V\right)\left[R, \rho^{\delta}\right] \Psi\right\|+\left\|\left[R, \rho^{\delta}\right]\left(\partial_{i} V\right) R \Psi\right\| .
$$

Thus this statement follows from a) and b).

Now we show the implication $(A 1) \Rightarrow(A 2)$.

Let's assume (A1). Then:

$$
\|R \Psi\|_{n+\delta}=\left\|\rho^{\delta} R \Psi\right\|_{n} \leqq\left\|R \rho^{\delta} \Psi\right\|_{n}+\left\|\left[\rho^{\delta}, R\right] \Psi\right\|_{n} .
$$

The first term is bounded on $L_{n}^{2}$ by hypothesis. In the second term we expand the commutator as in part a). By assumption $R, \partial_{i} R$ are bounded on $L_{n}^{2}$. Mimicking the proof of $\mathrm{c}$ ) one can show that $R \partial_{i}$ is also bounded. Moreover the multiplication by $\partial_{i} \rho^{\delta}$ is bounded $L_{n}^{2} \rightarrow L_{n}^{2}$. Thus:

$$
\left\|\left[\rho^{\delta}, R\right] \Psi\right\|_{n} \leqq \mathrm{const}\|\Psi\|_{n} \leqq \mathrm{const}\|\Psi\|_{n+\delta},
$$

i.e. the first conclusion in (A2) holds.

The proof of boundedness of $\partial_{i} R$ goes along the same lines. We write:

$$
\left\|\partial_{i} R \Psi\right\|_{n+\delta}=\left\|\rho^{\delta} \partial_{i} R \Psi\right\|_{n} \leqq\left\|\partial_{i} R \rho^{\delta} \Psi\right\|_{n}+\left\|\left[\rho^{\delta}, \partial_{i} R\right] \psi\right\|_{n}
$$

and expand the commutator on the r.h.s. as in b). Then the second conclusion in (A2) follows from the induction assumptions.

For our purposes we also need:

Lemma B. Under similar assumptions the operator:

$$
\begin{array}{r}
\left.\left(1+\mid \tau(\varepsilon) x+\sum_{k=1}^{K} \tau_{k}^{(1)}(\varepsilon) \xi_{k}^{(1)}+\sum_{k=1}^{L} \tau_{k}^{(2)}(\varepsilon) \xi_{k}^{(2)}\right) \mid\right)^{2+\delta}\left(h_{\varepsilon}(x)+z\right)^{-1} \\
\left.\left(1+\mid \tau(\varepsilon) x+\sum_{k=1}^{K} \tau_{k}^{(1)}(\varepsilon) \xi_{k}^{(1)}+\sum_{k=1}^{L} \tau_{k}^{(2)}(\varepsilon) \xi_{k}^{(2)}\right) \mid\right)^{-2-\delta}
\end{array}
$$

where $h_{\varepsilon}(x)$ is given by (6), is bounded from $L_{\mu}^{2}$ to $L_{\mu}^{2}$ for arbitrary $\mu$.

Here we used the same notation as throughout the entire paper. Proof of this lemma can be obtained by mimicking the proof of Lemma A.

Acknowledgement. The author is greatly indebted to Professor George A. Hagedorn for suggesting the problem, his encouragement and many valuable comments.

\section{References}

1. Combes, J.M.: On the Born-Oppenheimer approximation. In: Araki, H. (ed.) International Symposium on Mathematical Problems in Theoretical Physics. Berlin, Heidelberg, New York: Springer, 1975, pp. 467-471

2. Combes, J.M.: The Born-Oppenheimer approximation. In: Thirring, W., Urban, P. (eds.) The Schrödinger Equation. Berlin, Heidelberg, New York: Springer, 1977, pp. 139-159 
3. Combes, J.M., Duclos, P., Seiler R.: The Born-Oppenheimer approximation. In: Velo, G., Wightman, A. (eds.) Rigorous Atomic and Molecular Physics. New York: Plenum, 1981, pp. 185-212

4. Combes, J.M., Seiler, R.: Regularity and asymptotic properties of the discrete spectrum of electronic hamiltonians. Int. J. Quant. Chem. XIV, 213-229 (1978)

5. Hagedorn, G.A.: Semiclassical quantum mechanics I: The $\hbar \rightarrow 0$ limit for coherent states. Commun. Math. Phys. 71, 77-93 (1980)

6. Hagedorn, G.A.: A time-dependent Born-Oppenheimer approximation. Commun. Math. Phys. 77, 1-19 (1980)

7. Hagedorn, G.A.: High order corrections to the time-dependent Born-Oppenheimer approximation I: Smooth potentials. Ann. Math. 124, 571-590 (1986)

8. Hagedorn, G.A.: High-order corrections to the time-independent Born-Oppenheimer approximation I: Smooth potentials. Ann. Inst. Henri Poincaré 47, 1-19 (1987)

9. Hagedorn, G.A.: High order corrections to the time-independent Born-Oppenheimer approximation II: Diatomic Coulomb systems. Commun. Math. Phys. 116, 23-44 (1988)

10. Hagedorn, G.A.: High order corrections to the time-dependent Born-Oppenheimer approximation III: Diatomic Coulomb systems. Commun. Math. Phys. 117, 387-403 (1988)

11. Klein, M., Martinez, A., Seiler, R., Wang, X.P.: On the Born-Oppenheimer expansion for polyatomic molecules. Commun. Math. Phys. 143, 607-639 (1992)

12. Klein, M., Martinez, A., Wang, X.P.: On the Born-Oppenheimer approximation of wave operators in molecular scattering theory. Commun. Math. Phys. 152, 73-95 (1993)

13. Reed, M., Simon, B.: Methods of Modern Mathematical Physics, Vol III. New York, San Francisco, London: Academic Press, 1979

14. Reed, M., Simon, B.: Methods of Modern Mathematical Physics, Vol IV. New York, San Francisco, London: Academic Press, 1979

Communicated by B. Simon 